\title{
Strangeness Production in Relativistic Heavy Ion Collisions
}

\author{
M. G. Munhoz \\ Departamento de Física Nuclear, Laboratório Pelletron, Instituto de Física, \\ Universidade de São Paulo, C.P. 66318, 5315-970 São Paulo, SP, Brasil
}

Received on 15 August, 2003.

\begin{abstract}
In this article, I'll present some of the motivations for the study of strangeness production in relativistic heavy ions collisions. Several experimental results have demonstrated that strangeness production is a powerful tool to investigate new phenomena in these collisions. Perspectives are still opened for new and exciting results in these studies.
\end{abstract}

\section{Introduction}

The aim of the experimental program of relativistic heavy ion collisions is to the study nuclear matter under extreme conditions of temperature and energy density. In order to achieve this goal, is necessary to understand the dynamics of such collisions. The most striking feature of relativistic heavy ion collisions is the copious production of particles of a great variety of species. The comprehension of the mechanisms of particle production is probably the most important endeavor in such studies. It is important to understand not only the abundance of particles produced, but all features they present, as phase space occupancy, correlations, collectivity and fluctuations. A special class of particles which production can reveal important features of relativistic heavy ion collisions are the strange particles. Strange mesons and barions are abundantly produced in these collisions. They have the special feature of introducing the production and the dynamics of strange quarks, not present in the original colliding nuclei. Besides that, strange quarks are the lightest, therefore easiest to produce quark after the up and down quarks.

The study of strangeness production has long been pointed as a possible signature for the occurrence of new phenomena in relativistic heavy ions collisions. The main novelty expected in such collisions is the formation of a Plasma of Quarks and Gluons (QGP), in which quarks and gluons are deconfined over an extended region [1,2]. Enhancement in strangeness production and the antihyperon to antibaryon ratio are two of the proposed signatures of QGP formation. The underlying physics is as follows.

In a deconfined QGP, strange quark pairs $(s \bar{s})$ can be copiously produced through gluon-gluon fusion $[3,4]$, while in a hadronic gas, $s \bar{s}$ pairs have to be produced via pairs of strange hadrons with higher production thresholds. Moreover, the time scale of the gluon-gluon fusion process is short, of the order of 1-3 $\mathrm{fm} \mathrm{c}^{-1}$. On the other hand, it is argued that the strangeness production rate in a chemically equilibrated hadronic gas might be as high as that in QGP. However, the time needed for a hadronic gas system to reach chemical equilibrium is significantly longer than the typical lifetime of a heavy-ion reaction of the order of $10 \mathrm{fm} \mathrm{c}^{-1}$.

In addition to enhanced production of strangeness, production of antihyperons should be further enhanced in QGP under finite baryon density. This can be seen in the simple Fermi energy-level picture. Low-energy levels of light quarks $(q)$ are already occupied by the excessive light quarks due to the finite baryon density; when the Fermi energies of light quarks are higher than the bare mass of an $s \bar{s}$ pair, $s \bar{s}$ pair production is energetically more favorable than that of $q \bar{q}$. Hence, the production of non-strange light antiquark is suppressed, resulting in a high $\bar{s} / \bar{q}$ ratio in QGP.

\section{Theoretical Approaches}

There are several approaches that try to explain the abundance and features of the particles produced in relativistic heavy ion collisions. We can place them in two major groups: microscopic and macroscopic approaches.

Macroscopic approaches correspond to the usage of thermal and hydrodynamic models to explain the particle production. The models require the assumption that the particles achieve local thermal and chemical equilibrium. In practice, the system will evolve as it expands and cools before finally freezing out as cold noninteracting hadronic matter. Strictly, what is measured in heavy-ion collisions is the set of abundances in the final stage, when hadronic finalstate interactions cease. However, the flavor composition of the system should not change much through hadronic finalstate interactions, and therefore the measured yields reflect the system at hadronization.

Initially, microscopic models were intended to describe nucleus-nucleus collisions based on a superposition of elementary hadronic interactions and thus provide a baseline, i.e., a description of what we would expect in a hadronic system without a QGP. The microscopic models, by taking out trivial effects such as the impact parameter dependence of an observable, and by describing the ordinary physics of nucleon-nucleon or nucleon-nucleus collisions, could allow us to recognize any emerging new physics in nucleusnucleus collisions. 

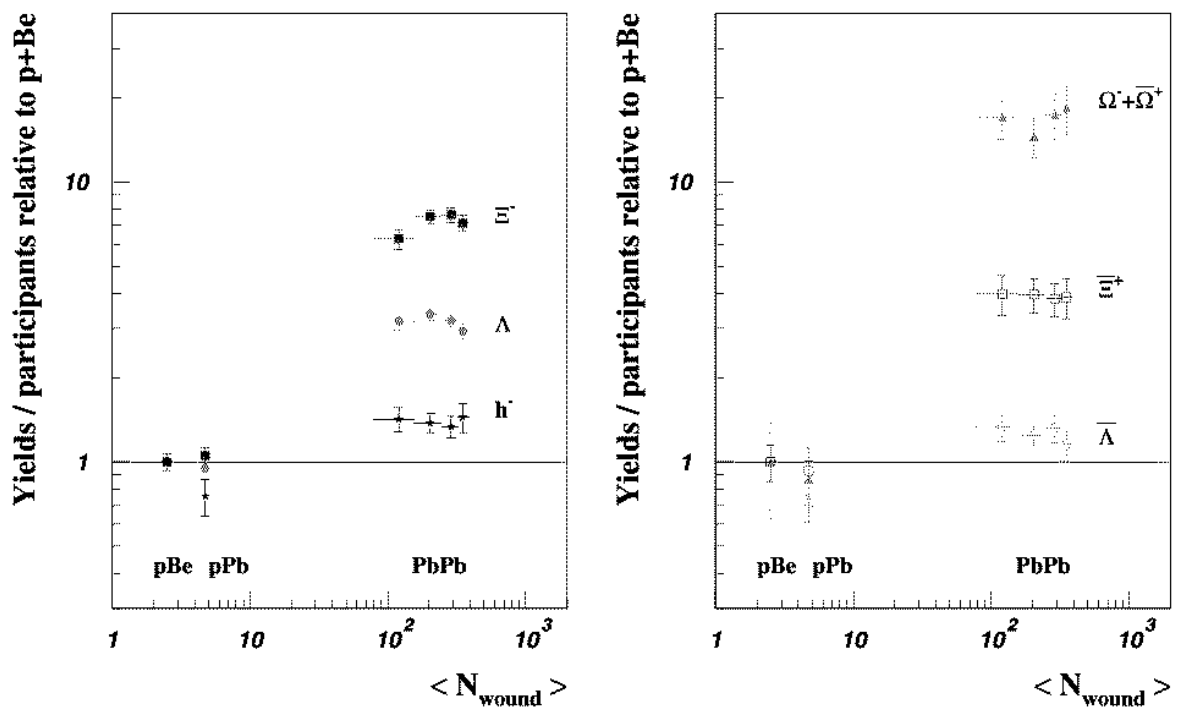

Figure 1. Yields per unit rapidity and per participant (i.e. wounded nucleon from Glauber) at central rapidity relative to $\mathrm{p}+\mathrm{Be}$ as a function of the number of wounded nucleons (WA97).
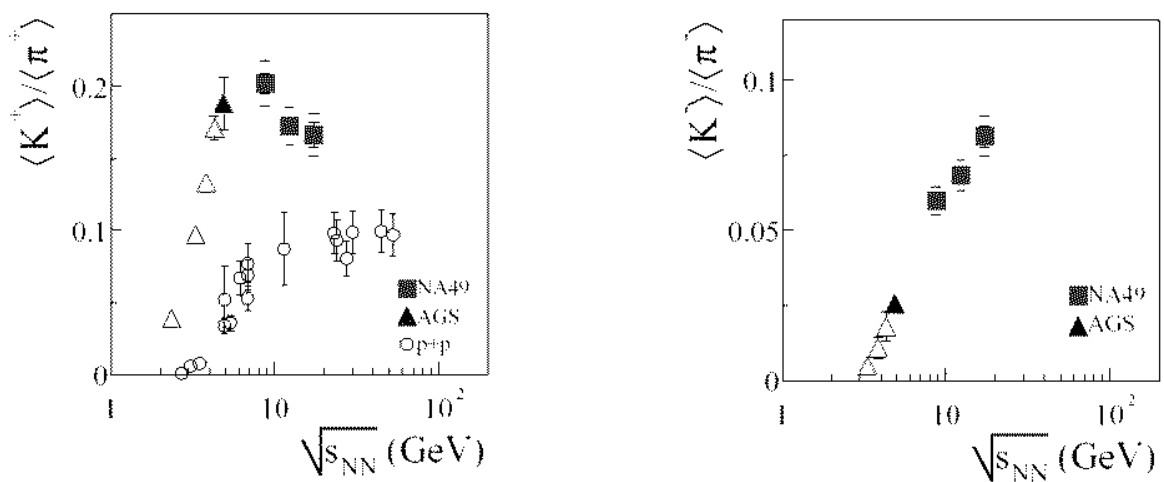

Figure 2. $K / \pi$ ratio for AGS and SPS energies in $\mathrm{A}+\mathrm{A}$ and $\mathrm{p}+\mathrm{p}$ collisions.

\section{Experimental Results}

\subsection{From AGS to SPS}

One of the most striking experimental result for strangeness production consists on the enhancement observed by the SPS experiments in $A+A$ collisions related to $p+p$ and $p+A$ collisions.

Figure 1 shows the results from experiment WA97 [5]. In this figure, the results are presented in the form of yields per unit of rapidity, centered at mid-rapidity and extrapolated down to zero $p_{t}$. It shows the yields of different particles per wounded (i.e. struck) nucleon as a function of the number of wounded nucleons, normalized to the values in $\mathrm{p}$ Be collisions. For each particle type, the yield per wounded nucleon are compatible between $\mathrm{p}-\mathrm{Be}$ and $\mathrm{p}-\mathrm{Pb}$ collisions. In $\mathrm{Pb}-\mathrm{Pb}$ collisions, they are also compatible for all measured centralities. However, they are enhanced with respect to the p-A yields by different factors, depending on the particle type.

Another experimental result that has challenged the the- oretical explanations is the ratio $K / \pi$, illustrated in Fig. 2 for AGS and SPS energies. As showed in the figure, the ratio $K^{+} / \pi^{+}$has a maximum value at $30 \mathrm{GeV} / \mathrm{A}$. A recent work [6] has shown that the statistical model description of relativistic heavy-ion collisions predicts the behavior presented by the data. They demonstrate that this maximum is due to the specific shape of the freeze-out curve in the $T-\mu_{B}$ plane. In particular, a very steep decrease of the baryon chemical potential with increasing energy causes a corresponding decline of relative strangeness content in thermal systems created in heavy-ion collisions above lab energies of $30 \mathrm{GeV}$. The saturation in T, necessary for this result, might be connected to the fact that hadronic temperatures cannot exceed the critical temperature $T_{c} \approx 170 \mathrm{MeV}$ for the phase transition to the QGP as found in solutions of QCD on the lattice. The maximum in the relative strangeness content is unique to heavy-ion collisions. There is no equivalent behavior in the collisions of elementary particles. 


\subsection{RHIC}

The latest result published by the STAR experiment is the measurement of the yields and the transverse momentum spectra of strange particles $(\Lambda, \Xi$ and $\Omega$ ) for $\mathrm{Au}+\mathrm{Au}$ collisions at $\sqrt{s_{N N}}=130 \mathrm{GeV}$ [7]. In this paper, is presented a fit to the $m_{t}$ spectra using a hydro-dynamically inspired function [8]. The model considers that all particles are emitted from a thermal expanding source with a transverse flow velocity $\left\langle\beta_{T}\right\rangle$ at the thermal freeze-out temperature $T_{f o}$. The solid lines of Fig. 3(a) show the one, two and three sigma contours for $T_{f o}$ vs $\left\langle\beta_{T}>\right.$ for the fit to the $\Xi^{-}$and $\Xi^{+}$data combined, with the diamond indicating the best fit result. Also shown, as the dashed lines, are the one, two and three sigma contours for a combined fit to the STAR $\pi, K$, $p$ and $\Lambda$ data, and the marker is the optimal fit location. The results for the two data sets do not overlap what might indicate that the $\Xi$ baryons show a different thermal freeze-out behavior than $\pi, K, p$ and $\Lambda$. Fig. 3(b) shows the mean $p_{T}$ for these particles calculated from the functions which best reproduce each $m_{t}$ spectrum. The band represents the model prediction based on the three sigma contour for the fit to the STAR $\pi, K, p$ and $\Lambda$ data while the lower dashed curve shows the prediction for the $T_{f o}=170 \mathrm{MeV},<\beta_{T}>=0$.
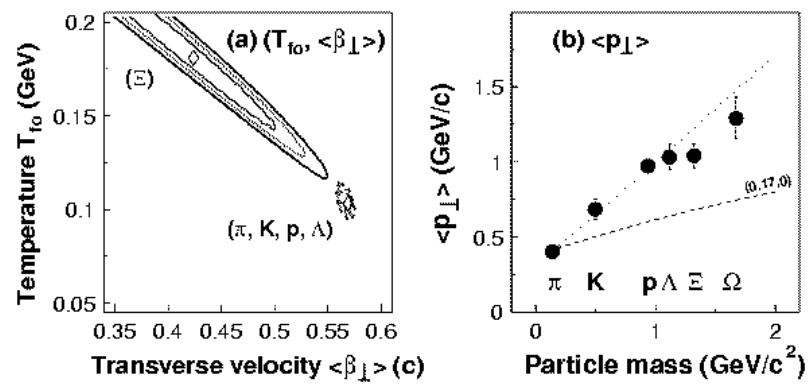

Figure 3. (a) The kinetic freeze-out temperature vs transverse flow velocity for the hydro-dynamically inspired model fits to the $m_{T}$ spectra [7]. The 1, 2 and 3 sigma contours are shown. Solid curves are for a simultaneous fit to the $\Xi^{-}$and $\bar{\Xi}^{+}$. Dashed curves are a separate fit to STAR $\pi, K, p$ and $\Lambda$ data. The diamonds represent the best fit in both cases. (b) Mean transverse momenta for identified particles vs particle mass (see text for details) [7]. The band results from the three sigma contour of the hydro-dynamically inspired model fit to the $\pi, K, p$ and $\Lambda$ data and the dashed curve is for $T_{f o}=170 \mathrm{MeV},<\beta_{T}>=0$.

Figure 4(a) shows the $\Xi^{-} / h^{-}$and $\bar{\Lambda} / h^{-}$ratios for the most central data. They increase from SPS energies to RHIC, whereas the $\Xi^{-} / h^{-}$ratio stays constant. This behavior indicates that the correlation between the increased strangeness production and reduction in the net-baryon number has to be considered. The proximity of the netbaryon number to zero at RHIC is reflected in the fact that the ratio of $\Xi^{-} / h^{-}$is close to $\Xi^{+} / h^{-}$. The reduction in the net-baryon number has a larger effect on the $\Lambda$ than on $\Xi^{-}$, as seen in Fig. 4(a), and thus creates the observed rise in the $\Xi^{-} / \Lambda$ ratio (Fig. $4(\mathrm{~b})$ ). The $\Xi^{+} / \bar{\Lambda}$ ratio is a constant from SPS to RHIC that might indicate that the scale of the multi-strange enhancement is the same for singly and doubly strange baryons.

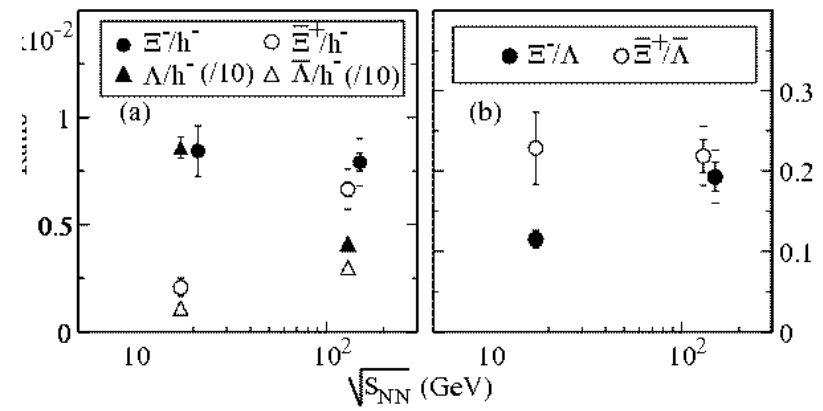

Figure 4. (a) $\Xi^{-}, \bar{\Xi}^{+}, \Lambda$ and $\bar{\Lambda}$ to $h^{-}$ratios and (b) $\Xi^{-} / \Lambda$ and $\Xi^{+} / \bar{\Lambda}$ ratios for the most central data as a function of $\sqrt{s_{N N}}$ [7]. The solid lines indicate the statistical uncertainty while the caps indicate the statistical and systematic uncertainties added in quadrature. Some ratios are slightly shifted along the $\mathrm{x}$-axis for clarity.

\section{Future Perspectives}

RHIC will have its next run in 2004. This period will allow the experimentalist to collect a large sample of events, increasing considerably the available statistics for strangeness production studies. A more detailed measurement, mainly, of $\Omega$ particles will allow us to reach further conclusions on very interesting questions as partonic flow.

\section{References}

[1] T.D. Lee and G.C. Wick, Phys. Rev. D 9, 2291 (1974).

[2] T.D. Lee, Rev. Mod. Phys. 47, 267 (1975).

[3] J. Rafelski and B. Muller, Phys. Rev. Lett. 48, 1066 (1982).

[4] J. Rafelski, B. Muller, and R. Koch, Phys. Rep. 142, 167 (1986).

[5] D. Rohrich, J. Phys. G 27, 355 (2001).

[6] P. Braun-Munzinger, J. Cleymans, H. Oeschler, and K. Redlich, Nucl. Phys. A 697, 902 (2002).

[7] J. Adams Lee et al. (2003), nucl-ex/0307024.

[8] E. Schnedermann, J. Sollfrank, and U. Heinz, Phys. Rev. C 48, 2462 (1993). 